\title{
Assessment of the Appropriateness of Ceftazidime Use in a Tertiary Teaching Hospital, Northern Ethiopia
}

This article was published in the following Dove Press journal: Drug, Healthcare and Patient Safety

\author{
Teklu Gebrehiwot \\ Gebremichael (D) ' \\ Hiluf Hindeya Gebreyesus ${ }^{2}$ \\ Alem Gebremariam ' \\ 'Clinical Pharmacy Unit, School of \\ Pharmacy, College of Health Sciences, \\ Mekelle University, Mekelle, Tigray, \\ Ethiopia; ${ }^{2}$ Medical Physiology Unit, \\ Biomedical Division, School of Medicine, \\ College of Health Sciences, Mekelle \\ University, Mekelle, Tigray, Ethiopia
}

Background: Ceftazidime is nowadays one of the most commonly used antibiotics due to its high antibacterial potency, wide spectrum of activity, and low potential for toxicity. However, the global trend shows huge misuse of ceftazidime.

Objective: This study was conducted to assess the appropriateness of ceftazidime use and to identify areas of intervention to prevent inappropriate use in different wards of Ayder Compressive Specialized Hospital, a tertiary teaching Hospital, Mekelle-Ethiopia.

Methods: A facility-based prospective cross-sectional study design was steered on 327 patients who received ceftazidime during their hospitalization in the selected wards from February 1 to April 30, 2019.

Results: In the assessment of the appropriateness of ceftazidime use, $2,084(70.8 \%)$ were appropriate. Appropriateness of indication was 295 (90.2\%), the effectiveness of ceftazidime use was $221(67.6 \%)$, correct dose of ceftazidime use was $264(80.4 \%)$, and the correct frequency of ceftazidime use was $230(70.3 \%)$. Its use was empiric in 275 participants $(84.1 \%)$ and specific in $52(15.9 \%)$ participants. The most common indication for ceftazidime use was uncomplicated pneumonia, at 112 (34.3\%). One hundred and seventy-one $(52.3 \%)$ participants had intervention to prevent inappropriate use of ceftazidime. Changing the drug combination $(96,29.4 \%)$, increasing the dose $(13,4 \%)$, decreasing the dose $(21$, $6.4 \%)$, holding the $(21,6.4 \%)$, and discontinuation of ceftazidime $(20,6.1 \%)$ were among the interventions

Conclusion: This study revealed that more than one-fourth of the ceftazidime use was inappropriate. This may lead to the emergence of resistant pathogens which in turn lead to treatment failure and increased the cost of therapy. Therefore, adherence to current evidencebased guidelines and initiating antimicrobial stewardship are recommended.

Keywords: ceftazidime, drug use evaluation, appropriateness, resistance

\section{Background}

Antibiotics are used to save the lives of millions of people and are among the most common medications prescribed in healthcare settings. ${ }^{1,3}$ Cephalosporin's are a commonly used class of antibiotics worldwide and the use of a newer generation of cephalosporin's have increased in the developing countries. The availability of various generations of cephalosporin like ceftazidime (3rd generation), cefalexin (1st generation), cefotaxime (3rd generation), ceftriaxone (3rd generation), cefixime (4th generation), and formulations as well as their expanded indications have an impact on prescribing and drug use practice. The spectrum of activities had been
Correspondence: Teklu Gebrehiwot Gebremichael

Clinical Pharmacy Unit, School of Pharmacy, College of Health Sciences, Mekelle University, Mekelle, Tigray, Ethiopia

Tel +25I-933-730-004

Fax +25I-34-4l6-68I

Email tekluu2020@gmail.com 
broadening in the third generation to include gram negatives like Enterobacter species, Klebsiella pneumoniae, and Pseudomonas aeruginosa. ${ }^{4,5}$

Third generation cephalosporins are the most commonly prescribed broad-spectrum antibiotic even before culture sensitivity test results arrive. Ceftazidime, which is a semisynthetic, broad-spectrum, beta-lactam antibacterial drug used for complicated and uncomplicated urinary infection, bone and joint infection, very severe life-threating infection in immune compromised patients, peritonitis, sepsis and serious genealogical and intra-abdominal infections, lower respiratory tract infections, skin and skin-structure infections, bacterial septicemia, central nervous system infections in different doses (250 mg, $500 \mathrm{mg}, 1 \mathrm{gm}, 2 \mathrm{gm}$ ) intravenous or intramuscular route of administration on BID (twice per day) and TID (three times per day) frequency. ${ }^{1}$

According to the World Health Organization (WHO), drug use evaluation (DUE) is a systematic, criteria-based drug evaluation that ensures the appropriateness of drugs. It is a method of obtaining information to identify problems related to drug use and means to correct the problem, thereby ensuring rational drug therapy. ${ }^{6,7}$ DUE of commonly used antibiotics will result in improved treatment efficacy, conserve costs, and prevent unwanted adverse effects. ${ }^{8,9}$ Irrational use of antibiotics can be associated with drug resistance, ${ }^{5}$ drug-induced diseases like adverse drug reactions, ${ }^{8}$ medicine stock outs, ${ }^{10}$ longer hospital stays, ${ }^{5}$ ineffective and unsafe treatment, prolongation of illness, distress, and higher costs. ${ }^{11}$

Developing resistance has been troublesome early after antibiotics became available for widespread use depending upon early reports of resistance in the literature or reports of healthcare transmission or outbreaks. ${ }^{12}$ The overuse of antibiotics had resulted in the advent of drug-resistant strains which are very difficult to treat, representing a major public health problem. ${ }^{13,14}$ Emergence of antibiotic resistant strains causes a considerable impact on patient treatment and outcomes, adding to the increased length of stay in the hospital, additional co-morbid conditions, and increased treatment cost. ${ }^{5}$ Many studies had been done on ceftriaxone use, but studies were lacking on the appropriateness of ceftazidime use. ${ }^{15,16}$ Since the status of ceftazidime use was not known in Ethiopia, this study was intended to assess ceftazidime use and to identify areas of intervention to prevent inappropriate use, which can be used as preliminary data for further research on drug resistance and sensitivity tests.

\section{Methods}

\section{Study Area}

This study was conducted in Ayder Comprehensive Specialized Hospital (ACSH), a tertiary and teaching hospital in Mekelle-Tigray, Northern Ethiopia, which is around $783 \mathrm{~km}$ from the capital city, Addis-Ababa. It started as a referral and specialized hospital in 2008 to a population of 8 million in the catchment areas of Tigray, Afar, and the South-eastern part of the Amhara regional states. It provides a broad range of medical services to both in-patient and outpatients. It also serves as a teaching hospital to several medical, dental, nursing, midwifery, public health, pharmacy, anesthesia, and medical laboratory students in both undergraduate and post-graduate programs. It is the second largest hospital in the nation, with more than 500 beds in the medical, pediatric, surgery, gynecology and obstetrics, adult and pediatric intensive care units. It has more than 100 specialists in various areas of medical specialization and more than 3,000 other health professionals.

\section{Study Design and Study Period}

A prospective cross-sectional study design was conducted from February 1 to April 30, 2019.

\section{Source and Study Population}

All patients admitted to the inpatient wards of ACSH for their medical care formed the study population. All patients admitted to medical, pediatric, intensive care unit (ICU), neonatal intensive care unit (NICU) and surgical wards of ACSH who took ceftazidime for their medical care during the study period were the study subjects.

\section{Eligible Criteria}

All patients admitted to inpatient wards of ACSH, who took ceftazidime within the study periods, were included in this study and outpatients were excluded from the study.

\section{Sample Size Determination and Sampling Technique}

The sample size was determined by using the single population proportion, assuming the prevalence rate of the good utilization of ceftazidime as $50 \%$, the desired degree of precision was $5 \%, 95 \%$ confidence interval.

$$
\mathrm{n}=\mathrm{Z}^{2} \alpha / 2 \mathrm{p}(1-\mathrm{p}) / \mathrm{d}^{2}
$$

where $\mathrm{n}=$ Required sample size; 
$\mathrm{z}=$ The standard score corresponding $95 \%$ confidence level $=1.96$;

$\mathrm{P}=$ Prevalence rate of good ceftazidime utilization $=50 \% ; \mathrm{d}=$ Margin of sampling error $=5 \%$; and

$\mathrm{n}=(1.96)^{2} \times 0.5 \times 0.5 /(0.05)^{2}=384.16, \sim 384$.

As the study population was less than 10,000 , around 15 participants were expected to take ceftazidime daily. Then $15 * 3 * 30=1,300$ finite population correction formulas were applied:

where $\mathrm{nf}=$ Desired sample size;

$\mathrm{n}=$ The calculated sample size;

$\mathrm{N}=$ Total population; and

$$
n f=\frac{384}{1+\left(\frac{n}{N}\right)} n f=\frac{384}{1+\left(\frac{384}{1300}\right)}
$$

$\mathrm{nf}=300$, with $10 \%$ non-response rate $=330$.

By adding a $10 \%$ (30) allowance to compensate for non-respondents and incompleteness, a total of 327 study subjects were recruited in this study. The study participants were selected by non-probability convenience or availability method where an attempt was done to include all participants who took ceftazidime during the study period without any probability sampling method until the required sample size for the study was obtained.

\section{Data Collection Instrument and Data Collection Procedure}

A data abstraction tool was prepared from different guidelines and research articles to include information about socio-demographic characteristics (sex, age, and residence), clinical characteristics (admitted ward, comorbidities, and combination of disease presentation), common indications of ceftazidime, type of treatment ceftazidime use (empirical or specific), dose and frequency of ceftazidime use, concomitant drugs used with ceftazidime, and appropriateness of ceftazidime use (disease-ceftazidime interaction (Yes/No), any drug interaction with ceftazidime (Yes/No), appropriateness of indication (Yes/No), effectiveness of ceftazidime use (Yes/No), correctness of ceftazidime dose and its frequency (Yes/No), least expensive alternativeness of ceftazidime use for the indication (Yes/ No), presence of unnecessary duplication therapy with other drugs (Yes/No), and acceptability of the duration of ceftazidime use (Yes/No). ${ }^{17}$ The Appropriateness of Ceftazidime use was calculated from the variables indicated in Table 1.

Appropriate use of ceftazidime was defined as using ceftazidime for the intended indication, when the recommended dose and frequency of ceftazidime was administrated, the optimal duration of treatment was given, disease presentation and co-administered drug had no interaction with ceftazidime, effectiveness for the prescribed illness, its least expensiveness as compared with other drugs used for the same indication as well as nonduplication therapy with other drugs. ${ }^{18,19}$

Empirical treatment of ceftazidime was also defined as when ceftazidime was prescribed initially before or without identification of ceftazidime culture-sensitive bacterial pathogens, whereas specific treatment was defined as when ceftazidime was prescribed after identification of ceftazidime culture sensitive bacterial pathogens. ${ }^{19}$

In this study assessment of the common intervention provided by the attending physician and clinical pharmacist working in the selected wards was described as changing the drug combination with ceftazidime, increasing or

Table I Appropriateness of Ceftazidime Use in the In-Patient Wards of Ayder Comprehensive Specialized Hospital (N=327)

\begin{tabular}{|l|l|l|}
\hline Variables & $\begin{array}{l}\text { Appropriate } \\
\text { (No Problem) }\end{array}$ & $\begin{array}{l}\text { Inappropriate } \\
\text { (There Is a Problem) }\end{array}$ \\
\hline Disease drug interaction with ceftazidime & $295(90.2 \%)$ & $32(9.8 \%)$ \\
Any drug interaction with ceftazidime & $249(76.1 \%)$ & $78(23.9 \%)$ \\
Appropriateness of indication of ceftazidime & $295(90.2 \%)$ & $32(9.8 \%)$ \\
Effectiveness of ceftazidime use for the indication & $221(67.6 \%)$ & $106(32.4 \%)$ \\
Correctness of the dose of ceftazidime use & $263(80.4 \%)$ & $64(19.6 \%)$ \\
Correctness of the frequency of ceftazidime use & $230(70.3 \%)$ & $97(29.7 \%)$ \\
Least expensive alternativeness of ceftazidime use compared to others of equal utility & $75(22.9 \%)$ & $252(77.1 \%)$ \\
Unnecessary duplication therapy with other drugs & $234(71.6 \%)$ & $93(28.4 \%)$ \\
Acceptance of the duration of ceftazidime therapy & $222(67.9 \%)$ & $105(32.1 \%)$ \\
Total & $2,084(70.8 \%)$ & $859(29.2 \%)$ \\
\hline
\end{tabular}


decreasing the dose of ceftazidime administered, holding or discontinuation of the dose of ceftazidime.

Data was collected from patient medication record chart scrutinized in order to pursue all ceftazidime use in the selected wards and data was collected by trained data collectors under the supervision of the investigator.

\section{Data Quality Assurance and Analysis Procedure}

Training of data collectors was given to acquire the basic skills necessary for data collection during the follow-up periods. Every activity was strictly followed by the principal investigator for the completeness of collected data. The data abstraction format was commented on by independent specialists in the respective areas of the study settings. Terms used were made clear and pre-tests were done in Mekelle General Hospital before starting the main study and then correction was made according to the review of the pre-tested data. The data abstraction format was prepared in the English language since the data collectors were professionals. All completed data abstraction format was examined for completeness and consistency during data management, analysis by the principal investigator, so that data was intensively cleaned before analysis and then coded, entered by using EpiData version 3.1, and analyzed using SPSS version 21. Simple descriptive statistical analysis, including percentage and frequency distribution, was used to describe ceftazidime use in inpatient wards.

\section{Results}

\section{Socio-Demographic and Clinical} Characteristics of Study Participants

A total of 327 participants were included in the present study. The gender distribution of participants was nearly similar, with $166(50.8 \%)$ males. Two hundred and thirty-eight (72.8\%) were from rural areas. Most participants were from medical wards $(177,54.1 \%)$, followed by 96 (29.4\%) participants from the pediatric ward, 21 (6.4\%) from the ICU, 17 (5.2\%) from the surgical ward, and $16(4.9 \%)$ from the NICU. From this study, 98 (30\%) of the participants had more than four comorbidities. Congested heart failure $(82,25.1 \%)$, surgical site infection $(74,22.6 \%)$, and diabetic mellitus $(36,11.0 \%)$ were the most common comorbidities. More than half of the participants $(180,55 \%)$ had one indication, and $112(34.3 \%)$ of them had two infectious disease presentations (Table 2).
Table 2 Socio-Demographic and Clinical Characteristics of Participants Taking Ceftazidime in In-Patient Wards of Ayder Comprehensive Specialized Hospital $(\mathrm{N}=327)$

\begin{tabular}{|c|c|c|c|}
\hline Variables & Categories & $\begin{array}{l}\text { Frequency } \\
\text { (N) }\end{array}$ & $\begin{array}{l}\text { Percent } \\
\text { (\%) }\end{array}$ \\
\hline Sex & $\begin{array}{l}\text { Male } \\
\text { Female }\end{array}$ & $\begin{array}{l}166 \\
161\end{array}$ & $\begin{array}{l}50.8 \\
49.2\end{array}$ \\
\hline Age & $\begin{array}{l}<5 \\
6-15 \\
16-30 \\
31-59 \\
>60\end{array}$ & $\begin{array}{l}86 \\
47 \\
65 \\
71 \\
58\end{array}$ & $\begin{array}{l}26.3 \\
14.4 \\
19.9 \\
21.7 \\
17.7\end{array}$ \\
\hline Admission ward & $\begin{array}{l}\text { Medical wards } \\
\text { Pediatric wards } \\
\text { Intensive care unit } \\
\text { Neonatal intensive care } \\
\text { unit (NICU) } \\
\text { Surgical ward }\end{array}$ & $\begin{array}{l}177 \\
96 \\
21 \\
16 \\
17\end{array}$ & $\begin{array}{l}54.1 \\
29.4 \\
6.4 \\
4.9 \\
\\
5.2\end{array}$ \\
\hline Resident & $\begin{array}{l}\text { Rural } \\
\text { Urban }\end{array}$ & $\begin{array}{l}238 \\
89\end{array}$ & $\begin{array}{l}72.8 \\
27.2\end{array}$ \\
\hline $\begin{array}{l}\text { Number of } \\
\text { comorbidities }\end{array}$ & $\begin{array}{l}0 \\
1 \\
2 \\
3 \\
4 \\
>4\end{array}$ & $\begin{array}{l}0 \\
86 \\
6 \\
70 \\
67 \\
98\end{array}$ & $\begin{array}{l}0 \\
26.3 \\
1.8 \\
21.4 \\
20.5 \\
30.0\end{array}$ \\
\hline $\begin{array}{l}\text { Types of } \\
\text { comorbidities }\end{array}$ & $\begin{array}{l}\text { Congested heart failure } \\
\text { Surgical site infection } \\
\text { Diabetic Mellitus } \\
\text { Retrovirus infection } \\
\text { Chronic kidney disease } \\
\text { Hypertension } \\
\text { Cancer } \\
\text { Others* }\end{array}$ & $\begin{array}{l}82 \\
74 \\
36 \\
33 \\
32 \\
20 \\
13 \\
37\end{array}$ & $\begin{array}{l}25.1 \\
22.6 \\
11.0 \\
10.1 \\
9.8 \\
6.1 \\
4.0 \\
11.3\end{array}$ \\
\hline $\begin{array}{l}\text { Combination of } \\
\text { infectious } \\
\text { disease } \\
\text { presentations }\end{array}$ & $\begin{array}{l}1 \\
2 \\
3 \\
4 \\
>4\end{array}$ & $\begin{array}{l}180 \\
112 \\
14 \\
7 \\
14\end{array}$ & $\begin{array}{l}55.0 \\
34.3 \\
4.3 \\
2.1 \\
4.3\end{array}$ \\
\hline
\end{tabular}

Notes: *Co-morbidities: Tuberculosis $(n=15)$, Stroke $(n=10)$, Pancytopenia $(n=4)$, Seizure $(n=4)$, Asthma $(n=2)$, Hypothyroidism $(n=2)$.

\section{Indications and Prescribing Pattern of Ceftazidime}

From this study the top three indications for ceftazidime use were hospital-acquired pneumonia (112, 34.3\%), Peritonitis and Sepsis (60, 18.3\%), and meningitis (44, $13.5 \%$ ) (Table 3). Descriptive analysis of ceftazidime use indicated that most prescriptions were issued for empirical therapy $(275,84.0 \%)$. It was also found that only $52(16 \%)$ 
Table 3 Indications of Ceftazidime Use in the In-Patient Wards of Ayder Comprehensive Specialized Hospital ( $N=327)$

\begin{tabular}{|l|l|l|}
\hline Indication of Ceftazidime & $\begin{array}{l}\text { Frequency } \\
\text { (N) }\end{array}$ & $\begin{array}{l}\text { Percent } \\
\text { (\%) }\end{array}$ \\
\hline Uncomplicated pneumonia & 112 & 34.3 \\
Peritonitis and Sepsis & 60 & 18.3 \\
Meningitis & 44 & 13.5 \\
Intra-abdominal infections & 25 & 7.6 \\
Very severe life threating infection in & 23 & 7 \\
immunocompromised patient & & \\
Lung infection or cystic fibrosis & 22 & 6.7 \\
Complicated urinary tract infection & 21 & 6.4 \\
Bone and joints infections & 7 & 2.2 \\
Neutropenic Fever & 7 & 2.2 \\
Uncomplicated urinary tract infection & 6 & 1.8 \\
\hline
\end{tabular}

participants received ceftazidime for the specific therapy (Figure 1).

\section{Type of treatment of ceftazidime use}

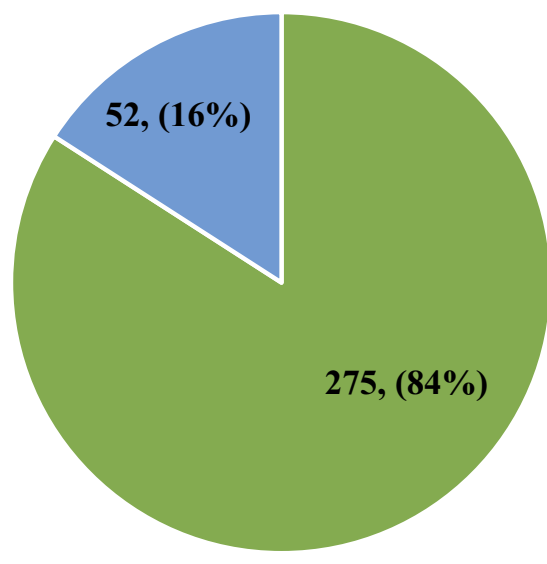

- Empirical

- Specific

Figure I Type of treatment of ceftazidime use in the inpatient wards of Ayder Comprehensive Specialized Hospital ( $\mathrm{N}=327)$.

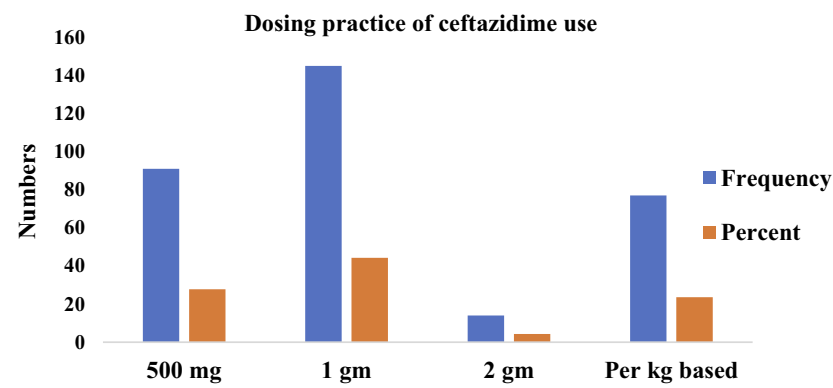

Figure 2 Dose of ceftazidime use in the inpatient wards of Ayder Comprehensive Specialized Hospital ( $\mathrm{N}=327)$ per kilogram based on $(30-50) \mathrm{mg} / \mathrm{kg}$ per dose in $<12$ years.

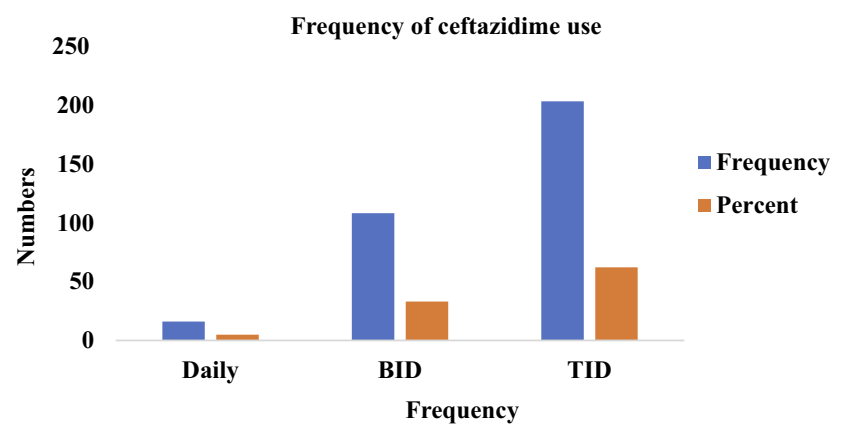

Figure 3 Frequency of ceftazidime use in the inpatient wards of Ayder Comprehensive Specialized Hospital (N=327), BID,Twice per day; TID, Three times per day.

\section{Dosage and Frequency of Ceftazidime}

The most commonly prescribed amount of ceftazidime per dose was found to be $1 \mathrm{gm}(145,44.3 \%)$ of participants. This was followed by the $500 \mathrm{mg}$ dose $(91,27.8 \%)$ of participants. The most common frequency of ceftazidime administration was found to be the TID (203, 62.1\%) of participants. The details of dosage and frequency of administration ceftazidime are shown in Figures 2 and 3.

\section{Concomitant Drugs Administrated with Ceftazidime}

Among the antibiotic drugs co-administered with ceftazidime, vancomycin $(208,63.6 \%)$ took the first place. This was followed by metronidazole $(67,20.5 \%)$. On the other hand, furosemide was the most common concomitant nonantibiotic drug used with ceftazidime (Tables 4 and 5).

\section{Appropriateness of Ceftazidime Use}

In this study, 2,084 (70.8\%) ceftazidime use was appropriate in the study participants. In the assessment of the appropriateness of ceftazidime use, 295 (90.2\%) of the participants

Table 4 Concomitant Antibiotic Drugs Administered with Ceftazidime in the In-Patient Wards of Ayder Comprehensive Specialized Hospital $(\mathrm{N}=327)$

\begin{tabular}{|l|l|l|}
\hline $\begin{array}{l}\text { Concomitant Antibiotic } \\
\text { Drugs }\end{array}$ & $\begin{array}{l}\text { Frequency } \\
\text { (N) }\end{array}$ & $\begin{array}{l}\text { Percent } \\
\text { (\%) }\end{array}$ \\
\hline Vancomycin & 208 & 63.6 \\
Metronidazole & 67 & 20.5 \\
Meropenem & 13 & 4.0 \\
Azithromycin & 13 & 4.0 \\
Ampicillin & 7 & 2.2 \\
Clindamycin & 7 & 2.1 \\
Ceftriaxone & 6 & 1.8 \\
Gentamycin & 6 & 1.8 \\
\hline
\end{tabular}


Table 5 Non-Antibiotics Concomitant Drugs Administered with Ceftazidime in the In-Patient Wards of Ayder Comprehensive Specialized Hospital $(\mathrm{N}=327)$

\begin{tabular}{|l|l|l|}
\hline $\begin{array}{l}\text { Concomitant Non-Antibiotic } \\
\text { Drugs }\end{array}$ & $\begin{array}{l}\text { Frequency } \\
\text { (N) }\end{array}$ & $\begin{array}{l}\text { Percent } \\
\text { (\%) }\end{array}$ \\
\hline Furosemide & 71 & 21.7 \\
Enalapril & 65 & 19.9 \\
Diclofenac & 41 & 12.5 \\
Tramadol & 32 & 9.8 \\
Insulin & 32 & 9.8 \\
3TC+TDF+EFV & 30 & 9.2 \\
Dexamethasone & 20 & 6.1 \\
Others* & 36 & 11.0 \\
\hline
\end{tabular}

Notes: * Morphine $(n=\mid 2)$, Aspirin $81 \mathrm{mg}(n=10)$, Phenobarbitone $(n=5)$, Warfarin $(n=4)$, Heparin $(n=3)$, L-thyroxine $(n=2), 3 T C=$ lamivudine.

Abbreviations: TDF, tenofovir disoproxil; EFV, Efavirenz.

had no disease that could interact with ceftazidime, there was no drug-drug interaction in 249 (76.1\%) participants, $295(90.2 \%)$ had appropriate indications, ceftazidime use was effective in $221(67.6 \%)$ participants, the correct dose of ceftazidime was used in 264 (80.4\%) participants, the correct frequency of ceftazidime was used in $230(70.3 \%)$ participants, and ceftazidime use was the least expensive alternative in 75 (22.9\%) participants. No unnecessary duplication of therapy with other drugs was seen in 234 (71.6\%) participants and the duration of therapy with ceftazidime was accepted in $222(67.9 \%)$ participants (Table 1$)$.

\section{Intervention Done in the Ceftazidime Use}

From this study 156 (47.7\%) participants were not given any intervention. But, 171 (52.3\%) of the participants had intervention on ceftazidime use. As shown in Figure 4, changing the drug combination (96, 29.4\%), increasing the

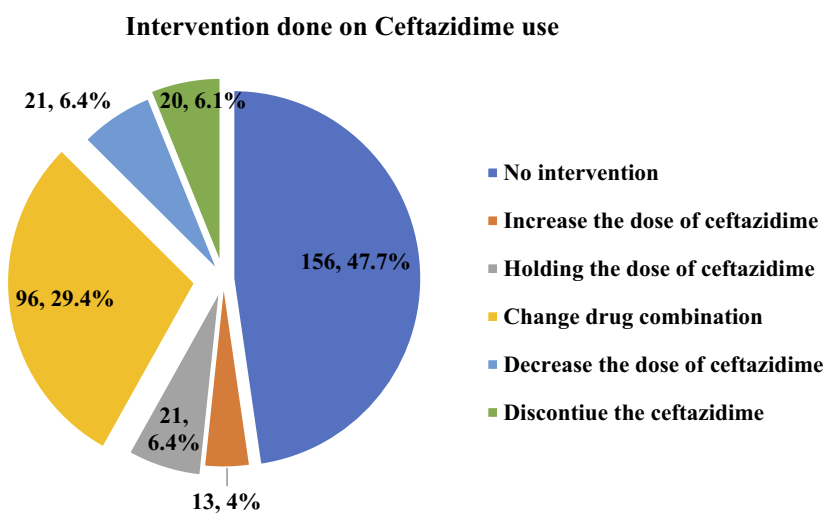

Figure 4 Intervention done on ceftazidime use in the inpatient wards of Ayder Comprehensive Specialized Hospital ( $N=327)$. dose of ceftazidime $(13,4 \%)$, decreasing the dose of ceftazidime $(21,6.4 \%)$, holding the dose of ceftazidime (21, 6.4\%), and discontinuation of the ceftazidime used $(20,6.1 \%)$ interventional changes were identified.

\section{Discussion}

This study was mainly aimed at evaluating the appropriateness of ceftazidime utilization by using nine criteria besides identifying the potential interventions recommended to prevent inappropriate use. Unlike with other antibiotics like ceftriaxone, few studies have been done on the appropriateness of ceftazidime use. ${ }^{15}$ The most prevalent primary indication for ceftazidime use in the current study was hospital-acquired pneumonia, supported by the study done in Eastern Eran. ${ }^{20}$ Vancomycin was the most common drug combination administered with ceftazidime (63.6\%) (from Table 4). This might be for better coverage in considering methicillin resistant staphaureous infections.

In this study, $84.1 \%$ ceftazidime use was for empirical treatment purposes, which shows a huge percentage of ceftazidime use was not based on the culture sensitivity test and there might be giant irrational prescribing practice. Prescribing empirically could be a risk for the development of resistance, increase costs, and compromise patient safety. Antimicrobial resistance is a serious growing public health problem and it is widely accepted that the major cause for the emergence and spread of antimicrobial resistance is misconsumption of antimicrobial drugs. Antibiotic prescribing guidelines should be considered before prescribing antibiotics like ceftazidime. ${ }^{18,19}$ Similarly, delayed ceftazidime prescribing strategies should also be introduced to reduce the misuse of ceftazidime.

In the assessment of the appropriateness of ceftazidime use from Table 1, being ineffective (32.4\%), the incorrect frequency (29.7\%), not the least expensive alternative drugs (77.1\%), having unnecessary duplication therapy (28.4\%), and non-optimal duration of therapy (32.1\%) of ceftazidime were factors that might be responsible in the development of antimicrobial resistance, and treatment failure to ceftazidime. This might be related with the more empiric use of ceftazidime in the study setting in contrast to undertaking culture and sensitivity tests. This was supported by a study done on the relationship between exposure to antibiotics and increased risk of infection with resistant pathogens at a population level ${ }^{14}$ with more pronounced to most antipseudomonal drugs like ceftazidime. ${ }^{21,22}$ In a study from the Slovak republic, $1.2 \%$ of Pseudomonas aeruginosa were resistant to meropenem, $4.1-7.7 \%$ to ceftazidime and cefepime, $12 \%$ to amikacin, 
and greater than $30 \%$ to ciprofloxacin. ${ }^{23,24}$ Therefore, providing training on the use of ceftazidime, preparing institutional prescribing guidelines of ceftazidime, and active involvement of a clinical pharmacist in each ward besides research on the resistant pattern and responsible factors for the resistance of ceftazidime are recommended.

In this study, the inappropriate indication of ceftazidime use was $9.8 \%$. This was comparably high as compared with the inappropriate indication of ceftriaxone use in Gonder $(3.5 \%)^{25}$ and very low as compared with that of ceftriaxone use in Tikur Anbessa specialized hospital $(18.5 \%){ }^{26}$ This might be due to duplicated therapy, or the presence of untreated indication. The presence of disease ceftazidime interaction might be attributed to the presence of chronic kidney diseases which need dose adjustment based on glomerular filtration rate. On the other hand, around one-quarter of the participants were on ceftazidime and furosemide, which might be a contributing factor for the drug-drug interactions identified in this study. Attention should be paid in prescribing furosemide or gentamycin with ceftazidime. Inappropriate dosing and the frequency of ceftazidime use might be related with poor professional communication and poor dose calculation based on the weight of the participants, especially in the pediatric wards and chronic kidney disease participants. ${ }^{27}$ On the other hand, since ceftazidime is given over 8 hours for most indications this might confuse the professional in changing from ceftriaxone to ceftazidime for more coverage. Giving attention to the right dose and right frequency of ceftazidime will help to prevent other inappropriate use. Proper dose adjustment and using online dosing information might also be recommended.

In the case of effectiveness of ceftazidime use, $32.4 \%$ of the participants were on ceftazidime for the intended indication. This might be due to the wrong dose and frequency of ceftazidime use, and drug interaction and inappropriate duration of therapy. Prolonged hospitalization might also be a factor that affects the effectiveness of ceftazidime.

In the current study, ceftazidime use was not the least expensive alternative in more than three-quarters of the participant's indication. Lack of knowledge on the available alternative drugs might be the leading reason. This could have a ignificant effect on the development of ceftazidime resistance in the study setting. Preparing appropriate firstand second-line alternative drugs for the intended indication might be helpful. Clinical conditions of the participant, length of hospitalization and professional knowledge as well as practice might be responsible for the non-optimal duration of ceftazidime use. Generally, in this study, the inappropriate use of ceftazidime was $29.2 \%$, unlike a study done in the United States, where $87 \%$ of ceftazidime use was inappropriate. ${ }^{15}$ The significant different in appropriateness of ceftazidime use might be due to the information gap between the studies.

During the study period, more than half of the participants had interventional treatment modifications on ceftazidime use. As shown in Figure 4, changing the drug combination had the highest percentage (29.4\%). This might be due to poor diagnosis and a lack of working guidelines on the specific use of ceftazidime in different indications. Among the other interventions holding the dose of ceftazidime and discontinuation of ceftazidime before the optimal duration was also high, which needs greater attention. This could be due to poor communication between professionals and weakness in regular follow-up of the clinical status of the participants. Therefore, improving knowledge and diagnostic confidence of healthcare professionals will be recommended.

\section{Limitations}

In this study, factors associated with inappropriate use of ceftazidime were not studied. Unlike with ceftriaxone use, studies were lacking on ceftazidime use, so a parallel comparison was not done with other study settings.

\section{Conclusions and Recommendations}

This study revealed that more than one-quarter of ceftazidime use was not appropriate. The least expensive alternativeness of ceftazidime had the highest degree of inappropriateness, followed by less effectiveness of ceftazidime use, unacceptable duration of ceftazidime therapy, unnecessary duplication therapy, incorrect frequency use, having drug interactions, whereas the appropriateness of indication of ceftazidime use was comparably good. More than half of the participants had one or more intervention on ceftazidime use during the study, noticeable by changing the combination of drugs, holding the dose of ceftazidime, and increasing the dose of ceftazidime. Prescribers should limit the use of ceftazidime only for infections proven or strongly suspected to be caused by ceftazidime susceptive microorganisms. As part of this, prescribers should also straight therapy with culture and sensitivity test result whenever it is possible.

Institutional policies should be prepared to reduce the inappropriate use of ceftazidime. Some policies may focus 
on improving the quality of the microbiology laboratory, establishing an antimicrobial stewardship program, consulting clinical pharmacists, and establishing institutional prescribing guidelines for antibiotics. Furthermore, research should be done to investigate factors attributable to high inappropriate use of ceftazidime and resistance pattern of ceftazidime in the study area.

\section{Abbreviations}

ACSH, Ayder Compressive Specialized Hospital; BID, Twice per day; ICU, Intensive care unit; NICU, Neonatal intensive care unit; TID, Three times per day.

\section{Operational Definitions}

Appropriate: The indication for use, dose, and frequency of administration, duration of treatment, culture and sensitivity test investigation, and drug-drug interaction with ceftazidime are according to the recommendations in the current treatment guidelines clinical and laboratory standards Institute. ${ }^{15}$

Inappropriate: The indication for use, dose, and frequency of administration, duration of treatment, and drug-drug interaction with ceftazidime was not according to the recommendations of the clinical and laboratory standards institute. ${ }^{15}$

Empirical treatment: Ceftazidime administration initiated before or without identification of ceftazidimesensitive bacterial pathogens. ${ }^{15}$

Specific treatment: Ceftazidime administration initiated after identification of ceftazidime-sensitive bacterial pathogens. $^{15}$

\section{Ethical Statement}

The institutional review board of the School of Pharmacy, College of Health Sciences, Mekelle University approved this study and the verbal informed consent. Verbal informed consent was obtained from each participant as well as parents or guardians for those children less than 18 years old. Confidentiality and privacy were maintained by using code during data collection. A letter of permission was also obtained from the chief clinical director of the ACSH. All the study participants were informed about the purpose of the study.

\section{Data Sharing Statement}

The datasets used and/or analyzed during the current study are available from the corresponding author on reasonable request.

\section{Acknowledgments}

We would like to thank Mekelle University, College Health Sciences for giving us this chance. Last but not least, we would like to thank all study participants and data collectors for their contribution to the success of our work.

\section{Author Contributions}

TGG and AG were involved in the conception, reviewing, analysis, study design, and wrote the manuscript. TGG, AG, and HHG participated in the study design, supervised the development of the manuscript, and were involved in manuscript writing and editing. All authors gave final approval of the version to be published and agreed to be accountable for all aspects of the work in ensuring that questions related to the accuracy or integrity of any part of the work are appropriately investigated and resolved.

\section{Funding}

The study was not supported by any funding agent.

\section{Disclosure}

The authors report no competing interests.

\section{References}

1. Mahalli A. WHO/INRUD drug prescribing indicators at primary health care centers in Eastern province, Saudi Arabia. EMHJ. 2012;18(11):1091-1095. doi:10.26719/2012.18.11.1091

2. Kohanski M, Dwyer D, Collins J. How antibiotics kill bacteria: from targets to networks. Nat Rev Microbiol. 2010;8(6):423-435. doi: $10.1038 /$ nrmicro2333

3. Gerber J, Newland J, Coffin S, et al. Variability in antibiotic use at children's hospitals. Pediatrics. 2010;126(6):1067-1073. doi:10.1542/ peds.2010-1275

4. Rice L, Willey S, Papanicolaou G, et al. Outbreak of ceftazidime resistance caused by extended-spectrum beta-lactamases at a Massachusetts chronic-care facility. Antimicrob Agents Chemother. 1990;34(11):2193-2199. doi:10.1128/AAC.34.11.2193

5. Bhartiy S, Shinde M, Nandeshwar S, Tiwari S. Pattern of prescribing practices in the Madhya Pradesh. KUMJ. 2008;6(1):55-59.

6. World Health Organization. Introduction to drug utilization research, World Health Organization, 20 Avenue Appia, 1211 Geneva 27, 2003. Available from: http://apps.who.int/medicinedocs/en/d/ Js4876e/. Accessed December 9, 2019.

7. Kaliamoorthy K, Sankaralingam R, Punniyakotti S, Janardhan V, Cheekala U. Drug utilization evaluation of third generation cephalosporin's. Pak J Pharm Sci. 2012;25(2):339-342.

8. Karande S, Sankhe P, Kulkarni M. Patterns of prescription and drug dispensing. Indian J Pediatr. 2005;72(2):117-121. doi:10.1007/ BF02760693

9. Shamshy K, Begum M, Perumal P. Drug utilization of antimicrobial drug in pediatrics population in a tertiary care hospital in Erode, Tamilnadu, India. Int J Pharm Tech Res 2011;3(3):1530-1536.

10. Cagri B, Ermertcan S, Hosgor-Limoncu M, Ciceklioglu M, Eren S. Rational antibiotic use and academic staff. Int $J$ Antimicrob Agents. 2003;21(1):63-66. doi:10.1016/S0924-8579(02)00272-8 
11. Vries T, Henning R, Hogerzeil H, Fresle D. Guide to Good Prescribing. Geneva, Switzerland: WHO; 1994.

12. Ventola C. The antibiotic resistance crisis. Part 1: causes and threats. $P \& T$ 2015;40(4):277-283.

13. Hogerzeil H. Promoting rational prescribing: an international perspective. Br J Clin Pharmacol. 1995;39(1):1-6. doi:10.1111/ j.1365-2125.1995.tb04402.x

14. Kucukates E. Antimicrobial resistance among gram-negative bacteria isolated from intensive care units in a cardiology institute in Istanbul, Turkey. Jpn J Infect Dis. 2005;58(4):228-231.

15. Okpara A, Van D, Cate T, Cheung L, Galley M. Concurrent ceftazidime drug use evaluation with clinical pharmacy intervention. Hosp Formul. 1994;29(5):392-4, 399, 402-4.

16. Davey P, Brown E, Fenelon L, et al. Systematic review of antimicrobial drug prescribing in hospitals. Emerg Infect Dis. 2006;12 (2):211-216. doi:10.3201/eid1202.050145

17. Tobgay T, Rai M, Aagaard-Hansen J, Johansen MV, Hansen EH, Trap B. Prescribing pattern of higher generation antibiotics in the out-patient setting in Bhutan hospitals. Asian Biomed. 2010;4 (2):349-353. doi:10.2478/abm-2010-0043

18. STG. Ethiopia Standard Treatment Guide Line. Healthcare Administration and Control Authority of Ethiopia; 2016.

19. CLSI. Performance Standards for Antimicrobial Susceptibility Testing.29th Ed. CLSI. Supplement M100. Wayne, PA: Clinical and Laboratory standards Institute; 2019.

20. Mohammd M, Mirrahimi B, Mousavi S, Moradi M. Drug use evaluation of three widely prescribed antibiotics in a teaching hospital in East of Iran. J Pharm Care. 2013;1(3):100-103.
21. Paramythiotou E, Lucet J, Timsit J, et al. Acquisition of multi drug-resistant pseudomona aeruginosa in patients in intensive care units: role of antibiotics with anti-pseudomonas activity. Clinical Infect Dis. 2004;38:670-677. doi:10.1086/381550

22. Cunha B. Pseudomonas aeruginosa: resistance and therapy. Semin Respir Infect. 2002;17(3):231-239. doi:10.1053/srin.2002. 34689

23. Lamia T, Bousselmi K, Saida B, Allah M. Epidemiological profile and antibiotic susceptibility of pseudomonas aeruginosa isolates within the burned patient hospitalized in the intensive care burn unit. Tunis Med. 2007;85(2):124-127.

24. Koprnova J, Beno P, Korcova J, et al. Bacteremia due to pseudomonas aeruginosa: results from a 3-year national study in the Slovak Republic. J Chemother. 2005;17(5):470-476. doi:10.1179/joc.2005. 17.5.470

25. Ayele A, Gebresillassie B, Erku D, et al. Prospective evaluation of ceftriaxone use in medical and emergency ward of Gonder university referral hospital. Pharmacol Res Perspect. 2018;6(1):e00383. doi:10.1002/prp2.383

26. Sileshi A, Tenna A, Feyissa M, Shibeshi W. Evaluation of ceftriaxone utilization in medical and emergency ward Tikur Anbessa specialized hospital. BMC Pharmacol Toxicol. 2016;17(7):6. doi:10.1186/ s40360-016-0057-x

27. Prajapati A, Ganguly B. Appropriateness of drug dose and frequency in patients with renal dysfunction in a tertiary care hospital: a cross-sectional study. J Pharm Bioallied Sci. 2013;5(2):136-140. doi:10.4103/0975-7406.111829
Drug, Healthcare and Patient Safety

\section{Publish your work in this journal}

Drug, Healthcare and Patient Safety is an international, peer-reviewed open-access journal exploring patient safety issues in the healthcare continuum from diagnostic and screening interventions through to treatment, drug therapy and surgery. The journal is characterized by the rapid reporting of reviews, original research, clinical, epidemiological and post-marketing surveillance studies, risk management, health

\section{Dovepress}

literacy and educational programs across all areas of healthcare delivery. The manuscript management system is completely online and includes a very quick and fair peer-review system. Visit http://www.dovepress.com/testimonials.php to read real quotes from published authors. 